\title{
Deconstructing Javanese Batik Motif When Traditional Heritage Meets Computation
}

\author{
Hokky Situngkir \\ [hs@compsoc.bandungfe.net] \\ Dept. Computational Sociology \\ Bandung Fe Institute
}

\begin{abstract}
The paper discusses some aspects of Iterated Function System while referring to some interesting point of view into Indonesian traditional batik. The deconstruction is delivered in our recognition of the Collage Theorem to find the affine transform of the iterated function system that attracts the iteration of drawing the dots into the complex motif of - or at least, having high similarity to - batik patterns. We employ and revisit the well-known Chaos Game to reconstruct after having some basic motifs is deconstructed. The reconstruction of the complex pattern opens a quest of creativity broadening the computationally generated batik exploiting its self-similarity properties. A challenge to meet the modern computational generative art with the traditional batik designs is expected to yield synergistically interesting results aesthetically. The paper concludes with two arrows of our further endeavors in this field, be it enriching our understanding of how human cognition has created such beautiful patterns and designs traditionally since ancient civilizations in our anthropological perspective while in the other hand providing us tool to the empowerment of batik as generative aesthetics by employment of computation.
\end{abstract}

Keywords: batik, fractal, attractor, iterated function system, affine transformation, culture anthropology, generative art 
Guided only by their feeling for symmetry, simplicity, and generality, and an indefinable sense of the fitness of things, creative mathematicians now, as in the past, are inspired by the art of mathematics rather than by any prospect of ultimate usefulness.

Bell, Eric Temple (1883-1960)

\section{Introduction}

As it has already been discussed in [10], traditionally Indonesian Batik has shown interesting fractal geometry while it has also been demonstrated that this property could broaden the acquisition of batik as model to generative art rooted from the traditional heritage. While we have recognized the steps of making the batik crafts [3], the computations of the innovative landscape of batik designs are now providing borderless creations of design applicable to enrichment the available traditional motif ${ }^{1}$. However, we can leave batik compositional design as an artificial generative art while we can delve deeper into the batik basic motif designs by deconstructing them.

We use the term "deconstruction" here by borrowing it from the terminology often used in philosophical or (post)-modern literary texts, roughly meant as finding meanings that were not originally intended by an author, composer, or artist of a cultural object (cf. [6]). Thus, here we use the terminology of batik deconstruction to denote a process decomposing a traditional motif in a way possibly different from the one used in the making and fabrication of the batik in general. As the purpose of the "deconstruction" in literary and philosophical is (perhaps) to understand the intangible things in the production of cultural object; here we incorporate the iterated function system and some conceptual related to it to understand the interesting self-similarity in the microsense of batik designs: the basic motif - a feature that is possibly an indirect intention when it is designed at the first place. While there have been some efforts to "deconstruct" traditional designs mathematically in some other places of ancient civilizations around the globe, batik is still left untouched without deep realizations in its mathematical aspects.

The batik process, represented by the word "mbatik" etymologically is realized most likely come from the Javanese phrase: "amba titik", meaning "drawing little dots". Here, the suffix "tik" could mean "little dot", "drop", or "point", however, it can also denote a ticking or trapping sound. In relation of batik designs with its function as fashion ornamentation, the root meaning of the suffix might also be seen in Javanese words such as "tritik" (a resist process by which designs are reserved on textiles by sewing and gathering before the dyeing process), or "nitik" (a design of batik imitating the weaving patterns). In short, we can always refer that mbatik is a representation of the drawing, painting, or writing [4]. Any drawings however, although not necessarily, would always be able to be understood elementarily as dotting. This is directly related to our further discussions on the iterated function system in the rest of the paper.

Iterative processes can be defined as repetitive steps that applied into the output of a system back as an initial state. Here, the output becomes the input and so on while the applied steps in the process do not have to same to all kinds of input but yet similar. The Iterated Function System regards the process with particular transformations that are applied repetitively with some geometric constraints yielding the self-similar patterns that we recognize as fractals. Thus, iterated function system is a way to have fractal geometry. The famous Multiple Reduction Copying Machine (MRCM) is an example to understand the iterated function system. MRCM regularly copies an image with some arrangements such that reducing the size of the origin while overlapping copies of the origin into the generated copy [8: 215-67].

\footnotetext{
${ }^{1}$ Some examples can be reviewed in: http: //fraktal. bandungfe. net
} 
The Iterated Function System is a system as a feedback loop in which the output of the previous copying process is used again as input of the next round of copying process. Interestingly, it does not matter with what picture or what points the input initially begin with, the resulting will eventually be "attracted" to certain fractal pattern. Thus, the problem of the deconstruction batik through the iterated function system is to reveal the attractors that made a fractal image as what it is.

The paper discusses basic motif of batik designs as the iterative drawing of dots emerging the wellknown pattern as we perceive. Here we already have a first step in deconstructing batik designs. We begin with discussion on model we use to generate fractal images, the Iterated Function System, the Barnsley's collage theorem [1], and some geometrical transformations incorporated composing fractal images. This is followed by the discussions on batik basic designs and the more likely affine transformation producing the designs. Eventually, we outline some conjectures and open problems to understand batik as a very interesting cognitive representation on aesthetics among Indonesian people, especially those with Javanese tradition. This however, broadens our endeavors to generally deconstructing batik.

\section{The Iterated Function System}

An iterated function system is a a system $w \in W$ where the set of affine transformations $w_{i}$, $i=1,2, \ldots, n$ mapping a subset of the plane $X \subset \Re^{2}$ onto the smaller subset $w_{i}(X)$ that can be written mathematically as transformation $W$ iteratively,

$W(X)=\bigcup_{i=1}^{n} w_{i}(X)$

The affine transformation $W$ can be written as a function of the mapping via $w$,

$$
w_{i}(X)=w_{i}\left(\begin{array}{l}
x \\
y
\end{array}\right)=\left(\begin{array}{ll}
a & b \\
c & d
\end{array}\right)\left(\begin{array}{l}
x \\
y
\end{array}\right)+\left(\begin{array}{l}
e \\
f
\end{array}\right)
$$

Thus, the affine transformations are governed by the coefficient of $a, b, c, d, e$, and $f$ and it easy to see that this transformation can be in the form of dilations,

$$
w_{\text {dilation }}(X)=\left(\begin{array}{ll}
r_{1} & 0 \\
0 & r_{2}
\end{array}\right)\left(\begin{array}{l}
x \\
y
\end{array}\right)
$$

where $r_{1}$ and $r_{2}$ the degree of contraction and stretching of $X$. It can also be a reflection,

$$
w_{r x}(X)=\left(\begin{array}{cc}
1 & 0 \\
0 & -1
\end{array}\right)\left(\begin{array}{l}
x \\
y
\end{array}\right) \text { and } w_{r y}(X)=\left(\begin{array}{cc}
-1 & 0 \\
0 & 1
\end{array}\right)\left(\begin{array}{l}
x \\
y
\end{array}\right)
$$

or translations that maps $w_{\text {translation }}(x, y)=(x+e, y+f)$,

$$
w_{\text {translation }}(X)=\left(\begin{array}{ll}
1 & 0 \\
0 & 1
\end{array}\right)\left(\begin{array}{l}
x \\
y
\end{array}\right)+\left(\begin{array}{l}
e \\
f
\end{array}\right)
$$


or rations that maps $w_{\text {rotation }}(x, y)=(x \cos \theta-y \sin \theta, x \sin \theta+y \cos \theta)$ respect to angle $0 \leq \theta \leq 2 \pi$,

$$
w_{\text {rotation }}(X)=\left(\begin{array}{cc}
\cos \theta & -\sin \theta \\
\sin \theta & \cos \theta
\end{array}\right)\left(\begin{array}{l}
x \\
y
\end{array}\right)
$$

or the shears or skews,

$$
\begin{aligned}
& w_{\text {sh1 }}(X)=\left(\begin{array}{ll}
1 & b \\
0 & 1
\end{array}\right)\left(\begin{array}{l}
x \\
y
\end{array}\right) \text { and } \\
& w_{\text {sh2 }}(X)=\left(\begin{array}{ll}
1 & 0 \\
c & 1
\end{array}\right)\left(\begin{array}{l}
x \\
y
\end{array}\right) .
\end{aligned}
$$

However, it is also possible to have the combination of the four, for instance the similitudes as the combination of dilation, translation, and rotation altogether, which can be written in the form

$$
\begin{aligned}
& w_{s 1}(X)=\left(\begin{array}{cc}
r \cos \theta & -r \sin \theta \\
r \sin \theta & -r \cos \theta
\end{array}\right)\left(\begin{array}{l}
x \\
y
\end{array}\right)+\left(\begin{array}{l}
e \\
f
\end{array}\right) \text { as well as, } \\
& w_{s 2}(X)=\left(\begin{array}{cc}
r \cos \theta & r \sin \theta \\
r \sin \theta & -r \cos \theta
\end{array}\right)\left(\begin{array}{l}
x \\
y
\end{array}\right)+\left(\begin{array}{l}
e \\
f
\end{array}\right)
\end{aligned}
$$

Thus, by using the homogenous coordinates, all of $w \in W$ are actually can be presented in the matrix form

$$
w_{i}([x, y, 1])=[x, y, 1]\left[\begin{array}{lll}
a & c & 0 \\
b & d & 0 \\
e & f & 1
\end{array}\right]
$$

or for the sake of simplicity

$$
w_{i}([x, y, 1])=[x, y, 1] F_{i}
$$

where $F_{i}=\left[\begin{array}{lll}a & c & 0 \\ b & d & 0 \\ e & f & 1\end{array}\right]$. The Chaos Game is defined with the probability of $p_{i}>0$ every time we have mapping of each $w_{i}$. All of them should thus fulfill

$$
\sum_{i=1}^{n} p_{i}=1
$$


The probabilities can be a uniform probability distribution, but the best one would be obtain by applying

$$
p_{i}=\frac{\left|\operatorname{det} F_{i}\right|}{\sum_{j=1}^{n}\left|\operatorname{det} F_{j}\right|}
$$

Computationally, the implementation of the Chaos Game can be conducted by applying the simple algorithm of ,

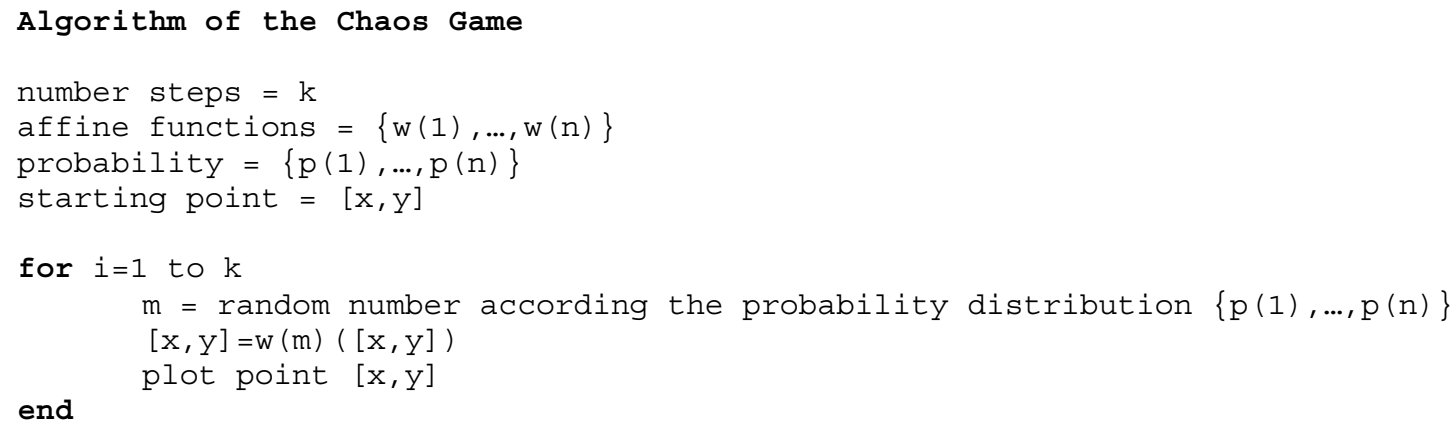

Thus, our deconstruction is to find the IFS and the Chaos Game that is associated to the basic motif of batik designs. In order to do this we hold on to the Collage Theorem as introduced in [1]. This theorem showed that a compact set of $A$ that we want to encode as the attractor of an iterated function system, we can discover parts of it that could resemble the whole. This is actually giving us a direct reminding of the properties of fractals: the self-similarity.

We tried to find the contractions $W$ such that each $W(A)$ is approximately a small piece of $A$. It is obvious that as we defined in equation (1), it should fulfill

$$
A \approx w_{1}(A) \cup w_{2}(A) \cup w_{3}(A) \cup \ldots \cup w_{N}(A) .
$$

Our approximation can be referred to the notion of Hausdorff distance [14]. We denote the contractivity factor $l<1$ for the iterated function system $W$, there is an attractor $A$ that is associated to our iterated function system,

$$
h(L, A) \leq \frac{\varepsilon}{1-l}
$$

Regarding to this equation, we could obviously realize that the best possible approximation can be yielded with the smallest possible contractivity factor $l$.

The discussions related to the deconstruction of two-dimensional objects are frequent in the field of image compression algorithm, known as fractal image compression [2]. Here, the detail of the compressed figures would be better with higher contractivity factor $l$. However, when we talk about deconstruction of the basic motif in batik designs, this constraint must also meet with what has been elaborated before in equation (1) where smaller $N$ is better; which means the simplest affine transformation that could reveal such complex and delicate objects of batik motif. This brings us to the challenging objectives in the deconstruction of batik as discussed in the rest sections. 
Table 1

The transformation of the basic motif of "Sawat"

\begin{tabular}{cccccccc}
\hline $\boldsymbol{w}$ & $\boldsymbol{a}$ & $\boldsymbol{b}$ & $\boldsymbol{c}$ & $\boldsymbol{d}$ & $\boldsymbol{e}$ & $\boldsymbol{f}$ & $\boldsymbol{p}$ \\
\hline $\mathbf{1}$ & 0.446 & 0.047 & -0.037 & 0.446 & 0.336 & 0.077 & 0.1747 \\
$\mathbf{2}$ & 0.694 & 0.129 & -0.088 & 0.763 & 0.141 & 0.062 & 0.471 \\
$\mathbf{3}$ & 0.063 & -0.307 & 0.129 & 0.12 & 0.483 & -0.027 & 0.0412 \\
$\mathbf{4}$ & 0.102 & -0.246 & 0.105 & 0.192 & 0.421 & 0.034 & 0.0396 \\
$\mathbf{5}$ & 0.21 & -0.115 & 0.051 & 0.39 & 0.34 & 0.111 & 0.0764 \\
$\mathbf{6}$ & 0.228 & 0.067 & -0.028 & 0.421 & 0.341 & 0.224 & 0.0851 \\
$\mathbf{7}$ & 0.250 & 0.168 & -0.072 & 0.465 & 0.380 & 0.278 & 0.1119 \\
\hline
\end{tabular}
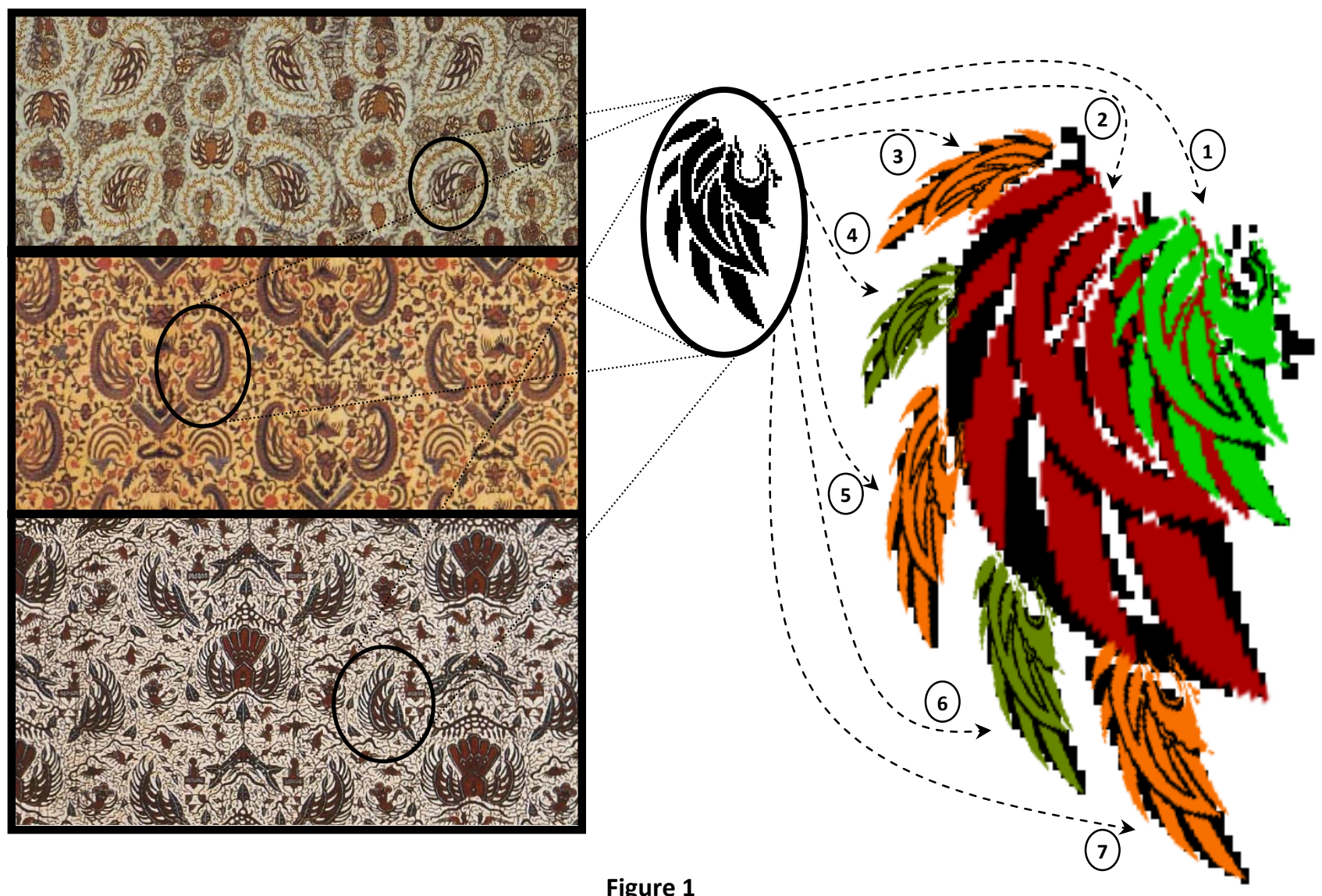

Figure 1

"Sawat", as elementary form of traditional batik exemplified here with designs of "Sawat Rinengga", "Semen Rama", "Semen Gurdha" [3: 140], with its corresponding Iterated Function System

\section{Batik in the terms of Iterated Function System}

There are several basic batik patterns are interestingly showed the pattern that can be deconstructed as some elements of transformations in the term of iterated function system. For instance, a traditional batik from Surakarta entitled "Sawat Rinengga" is interestingly can be seen as a result of seven transformation from its whole pattern as shown in figure 1 . In fact, as it shows in the figure, a lot of Javanese batik exhibits this basic pattern. The calculation of the linear equations of each transformation, $W$ (eq. 1 \& eq. 13 ) is listed in table 1 . Thus, we can easily write that the element of the Sawat Rinengga can be mathematically yielded by

$$
A^{\text {sawat }} \approx w_{1}\left(A^{\text {sawat }}\right) \cup \ldots \cup w_{7}\left(A^{\text {sawat }}\right)
$$


Table 2

The transformation of the basic motif: "Mega Mendung"

\begin{tabular}{rrrrrrrr}
\hline $\boldsymbol{w}$ & $\boldsymbol{a}$ & \multicolumn{1}{c}{$\boldsymbol{b}$} & \multicolumn{1}{c}{$\boldsymbol{c}$} & \multicolumn{1}{c}{$\boldsymbol{d}$} & $\boldsymbol{e}$ & \multicolumn{1}{c}{$\boldsymbol{f}$} & \multicolumn{1}{c}{$\boldsymbol{p}$} \\
\hline $\mathbf{1}$ & 0.585 & 0 & 0.02 & 0.499 & 0.031 & 0.105 & 0.3678 \\
$\mathbf{2}$ & 0.409 & 0 & -0.003 & 0.499 & 0.546 & 0.133 & 0.2571 \\
$\mathbf{3}$ & 0.257 & 0.033 & -0.031 & 0.256 & 0.307 & 0.11 & 0.0841 \\
$\mathbf{4}$ & 0.235 & -0.084 & 0.084 & 0.236 & 0.513 & 0.065 & 0.0786 \\
$\mathbf{5}$ & -0.509 & 0.129 & -0.016 & -0.328 & 0.752 & 0.396 & 0.2125 \\
\hline
\end{tabular}
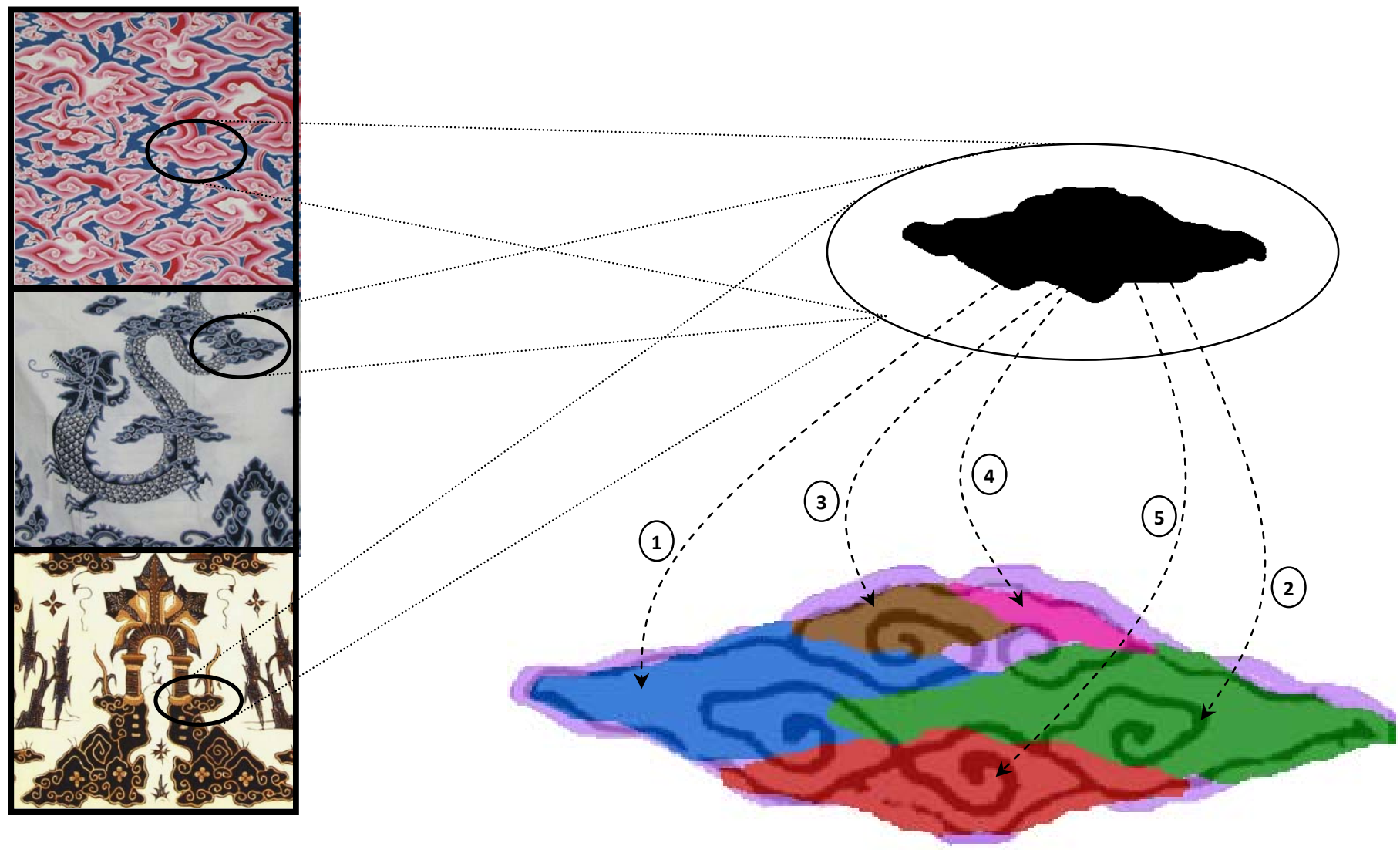

Figure 2

An elementary form of traditional, "Mega Mendung" with its corresponding Iterated Function System, also used in a great deal of batik designs from Cireon, West Java, Indonesia.

There are hundreds batik designs with this sawat ornamentation, since it is a drawing of the birds wing. A batik patterns such as semen, gurdha, and a lot more employ this famous basic pattern. Thus, it is interesting to found the general aspects related to the affine transformations used to emerge it. The other example can be taken from the Batik from Cirebon, West Java - Indonesia. A very well known design has motif called "Mega Mendung" which frequently becomes the basic ornamentation while it is recognized as stylization of "clouds in the sky". This is shown in figure 2.

In one hand, this accentuates a thing or two that traditional batik designs have incorporating the iterative function system. Here, the designer (a person that is traditionally recognized to be the intellectuals) made a kind of "affine transformations" of which (and definitely!) not mathematically. Thus, in the other hand, this can brought us to a lot more discussions related to more anthropological quests on batik, for batik designs, somehow can not be separated with the way of life and social fundamentals, in which they were designed, crafted, and appreciated. 
Regarding to the Collage Theorem (see eq. 14), the affine transformations, $w_{i}$, map any spatial input into the big picture of the pattern. The transformations attract the making of the designs to such pattern the designer want it to be. Thus, in our example above, sawat and mega mendung, as well other pattern of batik designs that can be regarded to be produced in the fashion iterated function system, is the attractor (cognitively) when an traditional artists would like to represent the "wings" and the "clouds". This could potentially enrich our understanding in cognitive anthropology while implemented into other collective and traditional aesthetics. For instance, as a Javanese traditional heritage, batik designs were actually cannot be separated with life in the king's palace (keraton). Nonetheless, in the ancient times, Javanese keraton had become the center of intellectuals as well as governance. The keraton's influence on batik designs thus showed the milestones of ancient intellectuals. For example, when the people at Cirebon, West Java, recognize two keratons (for any political reasons) i.e.: kasepuhan and kanoman (the older and the younger one respectively), both have characterizations of batik designs. The "cognitive attractors" on drawing the kingdom chariots, Keraton Kasepuhan drew the singa barong models while the Keraton Kanoman the Peksi Naga Liman [12]. This were also happens in the characteristic to other keratons in Indonesia such as Yogyakarta and Surakarta. What we begin here deconstructing Indonesian Javanese Batik could bring other challenges in understanding a lot of ancient social living of which now we recognized has inherently fractal properties.

In advance, some other interesting pattern that commonly found in Javanese traditional batik is elaborated in the next section in which we reverse the arrow of deconstructing by reconstructing batik basic designs. We do this by the acquisition of knowledge about the employment of some affine transformations to have the generated batik designs: a mode of computationally generative art.

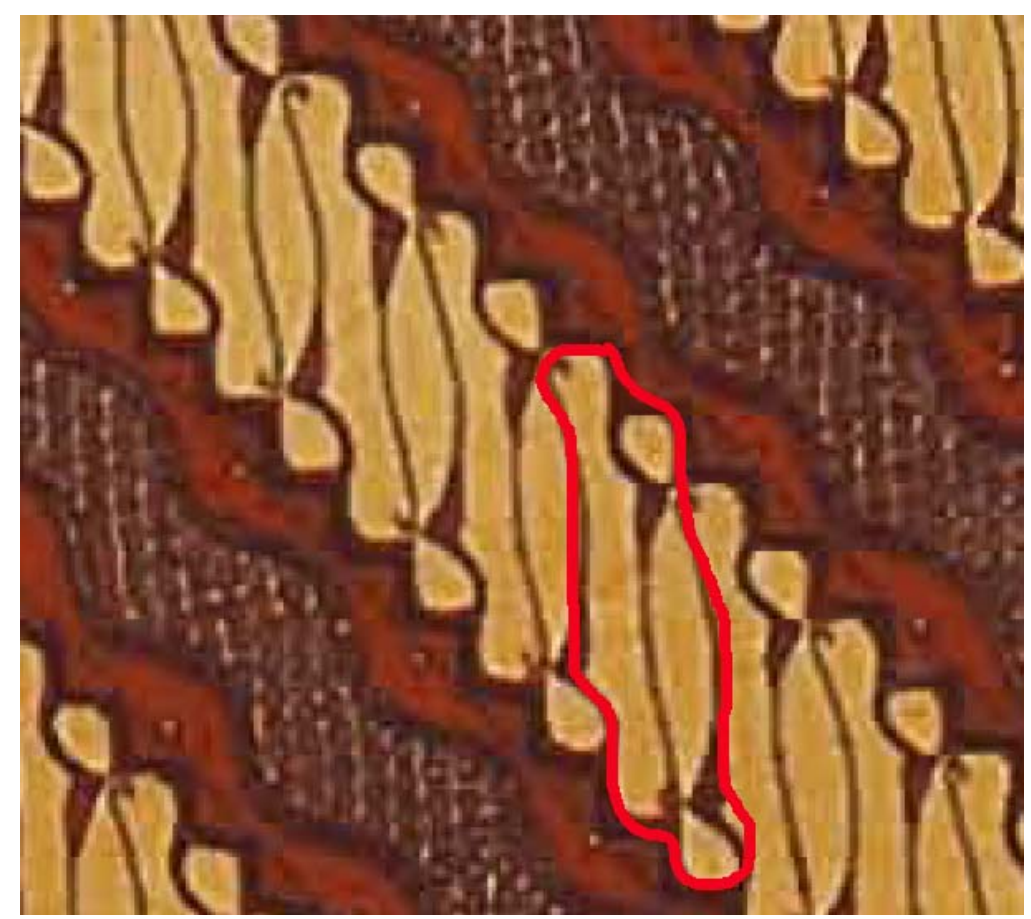

Figure 3

The attractor of Javanese "parang" motif. 


\section{Quest to Creativity: Generative Batik}

Beside the drawing that we have observed previously, some of batik patterns actually has shown a repetitive process in the making and can be easily recognized. For example is the well known motif parang. This is a very famous batik motif and there have also been a lot of batik patterns made by the innovation of the motif that is drawn in diagonal form runs parallel to each other repetitively. This motif have some disputed representation, for the word parang can be related to a "sword" (Malayan language) while there are also the old Javanese word referring it to "slope of canyon". The parang origins in unknown times and original designers, but some people traced it back to the times of Raden Panji, the hero from the $11^{\text {th }}$ century East Javanese Kingdom of Kediri and Jenggala [13]. Interestingly, the diagonal form of the motif can be observed as a set of the iterated function system's attractors as depicted in figure 3. Interestingly the Chaos Game applied to the attractor would emerging patterns that could lead to the repetitive style of the motif.

Understanding the iterated mechanisms on making the basic motif of batik reveals the elementary cognitive process on making the batik designs. As we have the model of the design-making of batik mathematically, then we have practically a great deal of motif stocks in our computational warehouse. An algorithm of "chaos game" as introduced in [1] can be incorporated computationally by applying the affine transformation with its respective probability. Chaos game is conducted by using randomly picked a point in the drawing-space as initial condition and then with certain probability (the seventh columns in our table 1 and 2). The resulting point is then dotted and becomes the input for the next iteration and so on. The dots would eventually emerges the pattern of which our affine transformations attract them. While we remember the etymological meaning of batik as "drawing dots", this becomes more interesting for the patterns we have from the Chaos Game are also emerged from the "attracted" dots.
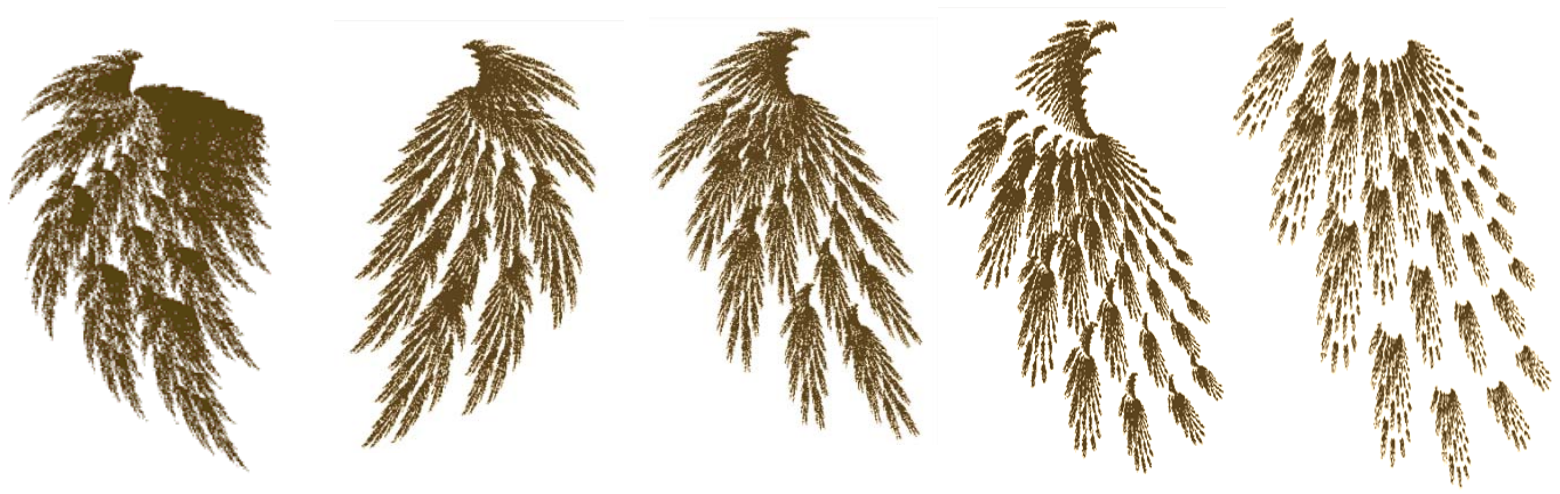

Figure 4

Some innovations from the Chaos Game Algorithm from simple and slight modifications of the sawat.

Moreover, as we have understood the deconstruction of the basic motif of batik in the fashion of affine transformation, then we can apply a great deal of other simple and beautiful result of iterated function system as a source for exploration in the innovation of batik pattern as well as creating new basic one. From the acquisition of the iterated function systems and the Chaos Game fractal models, we can therefore in the space of quest for creativity with computationally generated batik motif, at least by implementing these two applications:

\section{- Slight Modifications on the variables of the known batik affine transformations}

The sawat motif, for example, is now able to be produced in its various forms and pattern for changing the numbers in the matrix as showed in table 1 . We can imagine how many possible basic motif for batik designs that we can explore computationally, be it for the batik production or even 
for any other motif related creative fields. Related to the chaos and fractal theory, sometimes a simple and small changes on the coefficients could bring a lot of changes in the emerging pattern. Some of the patterns yielded in our arbitrary experiments on the sawat generative are presented in figure 4 . While batik is a traditional patterns with self-similarity aspects, this opens and even broader field for computational generated motif that can enrich batik models.

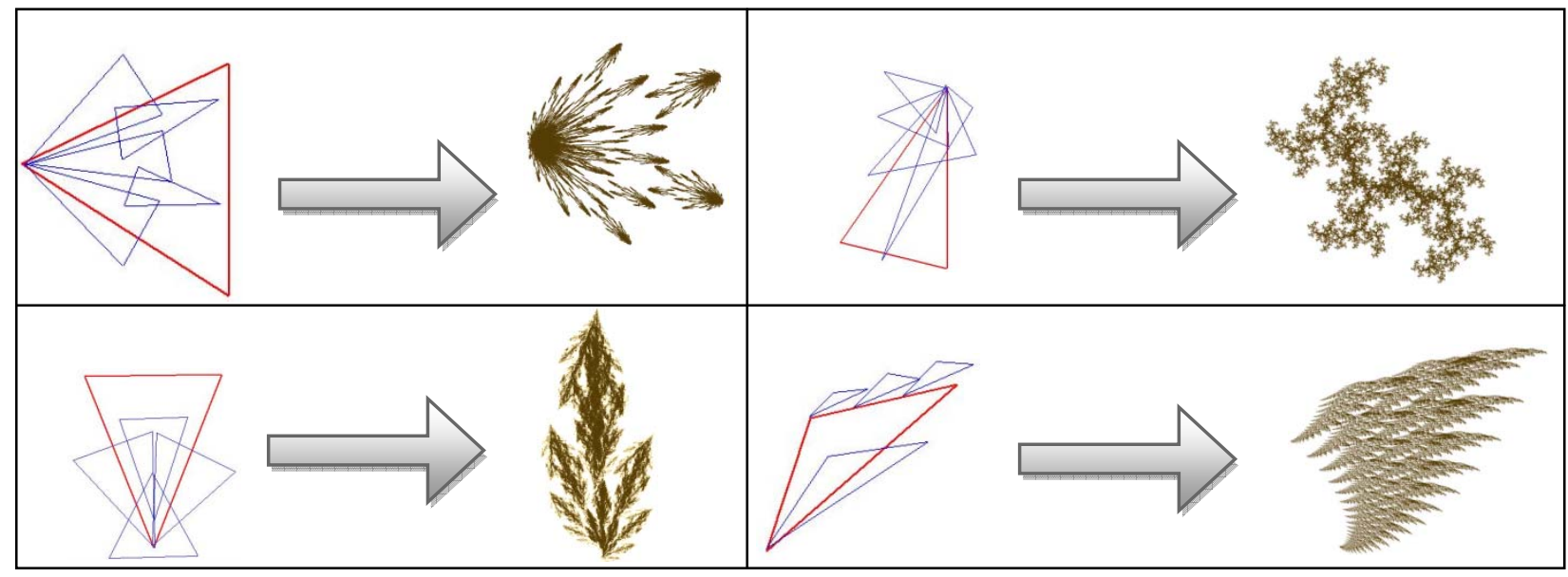

Figure 5

Example of several affine transformations in the iterated function systems that can be employed to broaden the batik generated motif.

- The incorporation of simple models of iterated function system in order to mimick things that can be applied as source for batik designs.

As it has been discussed in [1] as well as the discourse related to aesthetics and mathematics in [5] as well as [9], with more specific compositional designs of batik [10], when fractal mathematics can explain some generative process of beautiful patterns known in the domain of art, they can also be employed to inspire the new methods on generating art. Our Iterated Function Systems mimicking batik and the computational process of the Chaos Game thus can be used to inspire and broadened the study of batik as generative art. Some of the examples made from transformed triangles in elementary form is shown in figure 5. It is interesting to notice how the triangles can be eventually transformed into slightly different patterns. In some ways this is related to the Banach fixed-point theorem [7], that for considering the sequence of

$$
\begin{aligned}
& W^{0}(A)=A \\
& W^{n}(A)=W\left(W^{n-1}(A)\right), n \geq 1
\end{aligned}
$$

will be convergent to some $B$ in the same metric space, while $B$ will be independent on any choice of $A$.

Similar to those with the basic patterns in the deconstruction and the regenerating of original batik motif, the red-colored line triangles are the basic form approximations we would like to generate. The blue-lined triangles thus the self-similar triangles with affine transformations ( $w_{i} \in W$ ) from the red ones. Hence, in the figure 5, the two upper iterated function systems have $i=5$ and 4 , while the lower ones both have $i=4$. We can see an aspect of the complexity here that the simple forms of motif can be computationally emerging complex patterns - a point that we can get back to the possibly principles in batik as fractal, the complexity of batik designs that came from simple things 
yet become complex when they meet human cognition, be it the designing process as well as the appreciation.

Regarding to the elaboration of computationally generated batik primarily introduced in [10], compositional batik in this kind of generating process can be categorized as type one computationally generative batik, the fractals as batik. However, the fractal we used here is designed by deconstructing the traditional batik motif into affine transforms adhering the original one as the attractor in our iterated function system. This opens the limitless exploration into batik designs in our quest for creativity (cf. [11]).
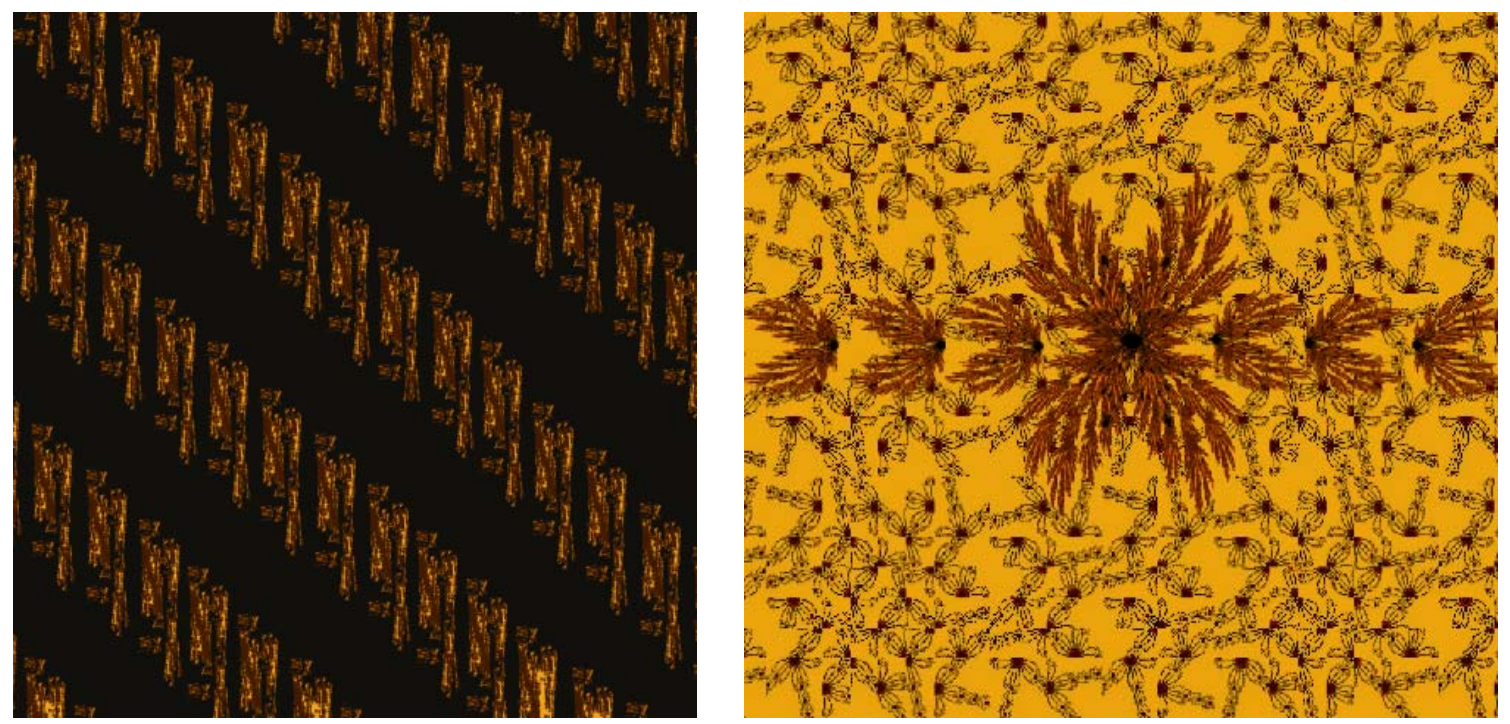

Figure 6

Two new designs computationally generated mimicking traditional batik: innovation on parang rusak (left), and innovation with sawat models with fillings using the fractal from Newton's method [10].

\section{Concluding Remarks}

We discussed the iterated function system and the Chaos Game when it is related to the hypothesis on the possibility of generating Indonesian traditional batik. By deconstructing some basic motifs of traditional batik, we associated them into the matrix representing affine transform, and in general the relation between the repetitive pattern in the making of batik motif with the iterated function system. It is exciting to remember that while Chaos Game is related to drawing dots repetitively in the affine transform, the word "batik" is rooted in the Javanese phrase of "drawing the dots".

The deconstruction of batik, in a way, opens new challenges on explaining a lot of complex motifs not only in the domain of Indonesian traditional heritage, but also in many other ancient civilizations exhibiting the similar fractal geometry. In a larger sense, this could be incorporated regarding to the approach of anthropological studies on the relation between culture and its artifacts with the evolving human cognition in the collective aspects throughout civilizations.

In the other hand, by deconstructing batik, we are also able to understand several aspects on how it emerges such fascinating patterns and in return gives us ability broadening the creativity for further batik aspects. We have seen some interesting aspects of the iterated system functions yielding great deal of complex patterns in the employment of computation of the Chaos Game algorithms. Simply speaking, this opens another door on the quest of creativity and aesthetics related to batik itself as well as our approaches to the generative arts in general. 


\section{Works Cited:}

[1] Barnsley. M. F. (1988). Fractals Everywhere. Academic Press.

[2] Barnsley, M.F. \& Hurd, L. P. (1993). Fractal Image Compression. AK Peters.

[3] Doellah, H. S. (2002). Batik: The Impact of Time and Environment. Danar Hadi.

[4] Fraser-Lu, S. (1986). Indonesian Batik: Processes, Patterns and Places. Oxford UP.

[5] Kappraff, J. (1991). Connections: The Geometric Bridge between Art and Science. McGraw-HII.

[6] Kigami, J., Strichartz, R. S., Walker, K. C. "Constructing a Laplacian on the Diamond Fractal". Experimental Mathematics 10 (3).

[7] Kirk, W. A., Khamsi, M. A. (2001). An Introduction to Metric Spaces and Fixed Point Theory. John Wiley.

[8] Peitgen, H-O, Jurgens, H., \& Saupe, D. (2004). Chaos \& Fractals: New Frontiers of Science $2^{\text {nd }}$ Ed. Springer.

[9] Situngkir, H. (2005). "What is the Relatedness of Mathematics and Art and Why We Should Care?". BFI Working Paper Series WPK2005.

[10] Situngkir, H. (2008a). "The computational generative patterns in Indonesian batik". BFI Working Paper Series WP-V-2008. URL: http : / www . bandungfe . net / ?go $=x p g \& \& c r p=48764471$

[11] Situngkir, H. (2008b). "Evolutionary Economics celebrates Innovation and Creativity based Economy". BFI Working Paper Series WP-X-2008. URL:

http : / / www . bandungfe. net / ? go=xpg\&\&crp=48d7d9ef

[12] Sondari, K. \& Yusmawati. (2000). Batik Pesisir. Cultural Media Development Project Departement of Education and Culture, Republic of Indonesia.

[13] Tirta, I. (1996). Batik: A Play of Light and Shades. Gaya Favorite Press.

[14] Wright, D. J. (1996). Dynamical Systems and Fractals Lecture Notes. Online Publication. URL: http://www. math.okstate.edu/mathdept/dynamics/lecnotes/lecnotes.html 